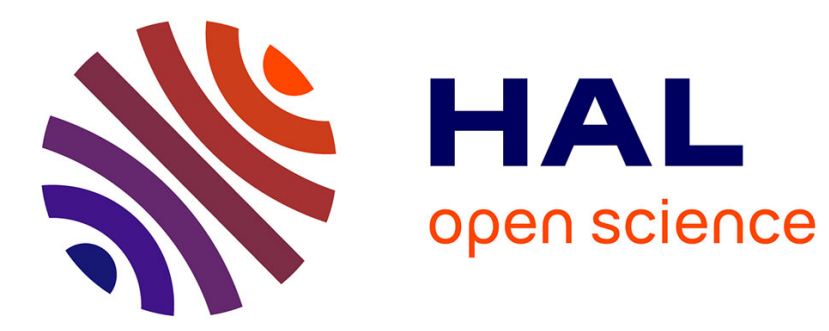

\title{
Modélisation expérimentale et numérique de milieux marins réels
}

\author{
G. Rabau, J. Piraux, J. Leandre, R. Holtzer
}

\section{To cite this version:}

G. Rabau, J. Piraux, J. Leandre, R. Holtzer. Modélisation expérimentale et numérique de milieux marins réels. Journal de Physique IV Proceedings, 1994, 04 (C5), pp.C5-1055-C5-1058. 10.1051/jp4:19945231 . jpa-00252918

\section{HAL Id: jpa-00252918 https://hal.science/jpa-00252918}

Submitted on 1 Jan 1994

HAL is a multi-disciplinary open access archive for the deposit and dissemination of scientific research documents, whether they are published or not. The documents may come from teaching and research institutions in France or abroad, or from public or private research centers.
L'archive ouverte pluridisciplinaire HAL, est destinée au dépôt et à la diffusion de documents scientifiques de niveau recherche, publiés ou non, émanant des établissements d'enseignement et de recherche français ou étrangers, des laboratoires publics ou privés. 


\title{
Modélisation expérimentale et numérique de milieux marins réels
}

\author{
G. RABAU, J. PIRAUX, J. LEANDRE et R. HOLTZER \\ CNRS, Laboratoire de Mécanique et d'Acoustique, 31 Ch. J. Aiguier, 13402 Marseille cedex 20, France
}

\begin{abstract}
In order to realize a complete geoacoustical model of the sea we have created a vertical variation in the sound velocity in a liquid layer presenting a minimum like in real sea by a diffusion technique in a tank. This technique involves the superimposing of several layers of miscible liquids with different densities without mixing. To simulate the sea bottom (with or without a $5 \%$ slope) we have used two types of material - Polyurethane resin to represent a sedimentary ground and marble to represent an elastic rock. To account for the scale, the measures were made at a frequency of $1 \mathrm{MHz}$ corresponding to $50 \mathrm{~Hz}$ in a real situation. The measured values are compared with the results of a numerical integration of the Helmholtz equation. We use a variant of the parabolic approximation.
\end{abstract}

\section{INTRODUCTION}

Notre but est de reproduire sur modèles réduits la propagation acoustique en milieu marin afin de valider les modèles théoriques ou numériques qui prennent en compte un profil vertical de célérité réel.

Par une technique de diffusion nous simulons, avec un facteur d'échelle de 1/20000, la propagation acoustique en mer à grande distance $(80 \mathrm{~km}$ ) et grande profondeur (de 0 à $4000 \mathrm{~m}$ ) en présence d'un chenal sonore sous-marin. La technique de diffusion [1,2] consiste à superposer en couches, sans les mélanger, plusieurs liquides miscibles de masses volumiques différentes (des couches d'eau alcoolisée, d'eau du réseau et d'eau salée). Des plaques en résine polyuréthane reproduisent un fond fluide sédimentaire et des plaques de marbre représentent un fond élastique rocheux [3]. Compte tenu du facteur d'échelle, les mesures sont effectuées à $1 \mathrm{MHz}$ ce qui correspond à $50 \mathrm{~Hz}$ en milieu réel. D'autre part la géométrie du fond, grâce à un jeu de caissons mobiles, peut être modifiée. On peut ainsi reproduire la transition petit fond - grand fond (passage d'un fond côtier au plateau continental) en installant une faible pente simulant la variation réelle entre ces deux types de fonds. Un banc perfectionné et entièrement automatisé permet de faire des mesures de propagation sonore en présence de ces différents éléments qui reproduisent le plus fidèlement possible les fonds marins réels. Les résultats de ces mesures sont comparés aux résultats de l'intégration numérique de l'équation de Helmholtz. On utilise la technique de l'équation parabolique en essayant de mettre en oeuvre ses perfectionnements les plus récents (approximation par fraction rationnelle de degré $\mathrm{N}$, méthode des directions alternées implicites, selfstarter ....).

\section{DESCRIPTION DU BANC EXPERIMENTAL}

Il s'agit d'installer ,en similitude, un profil vertical réel de célérité (profil TROP EST ETE 4000m) sur une hauteur de $20 \mathrm{~cm}$ (facteur d'échelle de 20000 ) dans une cuve expérimentale de $4,5 \mathrm{~m}$ de longueur, $0,88 \mathrm{~m}$ de largeur et $0,5 \mathrm{~m}$ de profondeur, implantée dans la cuve océanique du LMA.

Pour ce faire nous avons réalisé tous les équipements nécessaires à la mise en place dans de bonnes conditions des différentes couches de liquides, miscibles, de masses volumiques différentes, qui par diffusion lente vont établir dans la cuve expérimentale le profil vertical de célérité souhaité. 


\subsection{Bacs à aiguilles}

La technique de diffusion consiste à faire tomber en pluie les différents liquides dans l'ordre décroissant des masses volumiques. A partir du second liquide, pour éviter le brassage avec la couche inférieure, on place sur cette dernière un répartiteur flottant qui n'est autre qu'un film de mousse polyéther à cellules ouvertes. La "pluie" évoquée plus haut est obtenue à partir de 6 bacs parallélépipédiques en PVC plié et soudé de dimensions intérieures : longueur $0,99 \mathrm{~m}$, largeur $0,75 \mathrm{~m}$ et hauteur $0,115 \mathrm{~m}$. Sur le fond de chacun de ces bacs on a implanté 507 aiguilles de $1,2 \times 40 \mathrm{~mm}$ (ajutage de $0,8 \mathrm{~mm}$ de diamètre). L'alimentation en liquide de ces bacs est assurée par une station d'injection.

\subsection{Station d'injection contrôlée des liquides}

Cette station comprend essentiellement, une pompe, un compteur volumétrique programmable, un coffret électrique de commande et une électro-vanne d'alimentation des bacs à aiguilles. Cet ensemble permet d'injecter le volume de liquide nécessaire à chaque strate.

\subsection{Sources de pression acoustique et hydrophones de mesure}

Dans le but d'étudier la propagation des ondes acoustiques dans des strates liquides installées dans notre cuve expérimentale qui, rappelons le, réalisent après diffusion lente un profil vertical de célérité, nous utilisons à l'emission des transducteurs ULTRAN de la série W. Le modèle retenu WS 37 de fréquence centrale $1 \mathrm{MHz}$ a un élément actif de diamètre $9 \mathrm{~mm}$. Nous le faisons travailler en trains d'onde.

En réception nous utilisons un hydrophone "large bande" de notre fabrication . IL fonctionne à 1,5 MHz. La source et le récepteur sont placés sur un système mécanique qui permet des déplacements précis. Le controle des signaux émis, du déplacement des transducteurs et la mesure du niveau reçu par le récepteur sont assurés par un ordinateur. La mesure est entièrement automatique.

\subsection{Célérimètre}

Pour contrôler l'établissement du profil installé, nous avons réalisé un célérimètre simple et surtout peu encombrant pour perturber le moins possible les strates lors de son déplacement. Il est constitué de deux hydrophones "large bande", identiques à celui décrit plus haut maintenus dans un plan horizontal à une distance fixe de $13 \mathrm{~cm}$. Les mesures de célérité sont faites en trains d'onde à $3 \mathrm{MHz}$.

Tous ces appareillages font partie d'un ensemble automatisé. Cela nous permet de faire de nombreuses mesures avant la dégradation du profil de célérité installé.

\section{PROFIL VERTICAL DE CELERITE REALISE EN CUVE}

Le profil désiré proche d'un profil réel, à l'écelle $1 / 20000$ eme est obtenu après diffusion lente de 7 liquides miscibles installés en couches à partir de la surface sur une hauteur totale de $20 \mathrm{~cm}$. La mise en place des 7 couches de liquides, dont les caractéristiques sont données dans le tableau ci dessous, a été faite selon la procédure décrite plus haut.

tableau des liquides

\begin{tabular}{|c|c|c|c|}
\hline $\begin{array}{c}\mathbf{N}^{\circ} \text { des strates } \\
\text { épaisseur et volume }\end{array}$ & $\begin{array}{l}\text { Désignation détaillée } \\
\text { des liquides de base }\end{array}$ & $\begin{array}{l}\text { Masse volumique en } \\
\mathrm{g} / \mathrm{dm}^{3} \text { à } 20^{\circ} \mathrm{C}\end{array}$ & $\begin{array}{l}\text { Célérité du son en } \mathrm{m} / \mathrm{s} \text { à } \\
220^{\circ} \mathrm{C}\end{array}$ \\
\hline $\begin{array}{l}\text { (1) } \mathrm{e}=1 \mathrm{~cm} \\
\mathrm{~V}=40 \text { litres }\end{array}$ & $\begin{array}{l}\text { d'alcool Ethylique et } \\
\text { d'eau }\left(6^{\circ} \mathrm{GL}\right)\end{array}$ & 989 & 1521,1 \\
\hline $\begin{aligned}(2) \mathrm{e}=1,5 \mathrm{~cm} \\
\mathrm{~V}=60 \text { litres }\end{aligned}$ & $\begin{array}{l}\text { d'alcool Ethylique et } \\
\text { d'eau }\left(2,15^{\circ} \mathrm{GL}\right)\end{array}$ & 994 & 1496,9 \\
\hline (3) $\begin{aligned} \mathrm{c} & =6 \mathrm{~cm} \\
\mathrm{~V} & =240 \text { litres }\end{aligned}$ & eau du réseau & 998 & 1482,6 \\
\hline $\begin{array}{l}\text { (4) } \begin{array}{l}\mathrm{e}=3,5 \mathrm{~cm} \\
\mathrm{~V}=140 \text { litres }\end{array}\end{array}$ & $\begin{array}{c}\text { Solution à } 0,5 \% \text { pp de } \\
\mathrm{NaCl}\end{array}$ & 1002 & 1487,3 \\
\hline (5) $\begin{array}{l}\mathrm{e}=3 \mathrm{~cm} \\
\mathrm{~V}=120 \text { litres }\end{array}$ & $\begin{array}{c}\text { Solution à } 1,1 \% p p \text { de } \\
\mathrm{NaCl}\end{array}$ & 1006 & 1494,3 \\
\hline (6) $\begin{array}{l}\mathrm{e}=3 \mathrm{~cm} \\
\mathrm{~V}=120 \text { litres }\end{array}$ & $\begin{array}{c}\text { Solution à } 1,7 \% \text { pp de } \\
\mathrm{NaCl}\end{array}$ & 1010 & 1500,9 \\
\hline $\begin{array}{l}\text { (7) } \mathrm{e}=2 \mathrm{~cm} \\
\mathrm{~V}=80 \text { litres }\end{array}$ & $\begin{array}{c}\text { Solution à } 3,3 \% p p \text { de } \\
\mathrm{NaCl}\end{array}$ & 1021 & 1520,6 \\
\hline
\end{tabular}


Les 3 figures suivantes montrent l'évolution du profil injecté au cours du temps. On voit dans la figure 2 que la partie haute est rectiligne. Pour remédier à ce problème on asperge régulièrement la surface du milieu avec de l'alcool pure. Grâce à cette opération le profil installé reste utilisable pendant une quinzaine de jours.

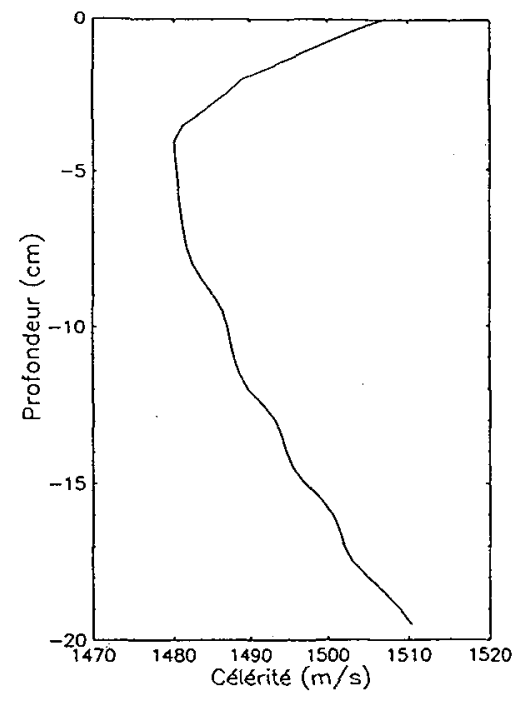

Figure 1 : Profil TROP EST ETE juste après son installation
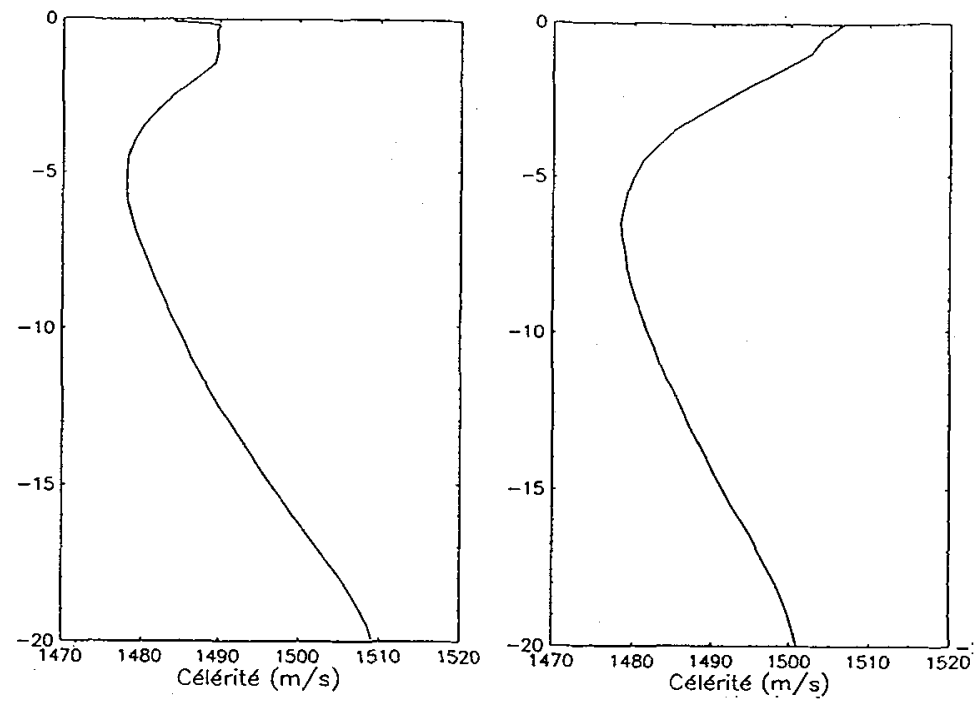

Figure 2: Profil TROP EST ETE

$12 \mathrm{H}$ après l'installation
Figure 3: Profil TROP EST ETE

$12 \mathrm{H}$ après l'installation avec pulvérisation d'alcool en surface

\section{MESURES ACOUSTIQUES ET COMPARAISON THEORIE-EXPERIENCE}

Les mesures suivantes sont faites avec un fond fluide, simulant un fond sédimentaire sablonneux réalisé en résines polyuréthanes dont la vitesse des ondes longitudinales $\mathrm{Cl}=1600 \mathrm{mS}-1$ (vitesse des ondes transversales nulle), ou avec un fond élastique simulant le socle rocheux réalisé avec des plaques de marbre de carrare de $3 \mathrm{~cm}$ d'épaisseur dont la vitesse des ondes longitudinales $\mathrm{Cl}=6017 \mathrm{mS}-1$. Les dalles de produit synthétiques reproduisant les différents fonds sont posées sur le fond de la cuve à plat ou sur un ensemble de caisson qui nous permettent de simuler une pente de $5 \%$ proche de celle reliant la zone cotière au plateaux continental.

Pour cette étude nous avons placé la source de pression à trois profondeurs d'immersion qui compte tenu du facteur d'échelle, correspondent à des profondeurs d'immersion en mer de 200,1000 et 2000 mètres.

Le tableau ci-après donne les profondeurs et les fréquences, réelles et transposées.

\begin{tabular}{|c|c|c|c|}
\hline \multicolumn{3}{|c|}{ Profil TEE $4000 \mathrm{~m}$ installé en similitude sur 20 cm de hauteur : } \\
Facteur d'échelle =20 000 \\
\hline $\begin{array}{c}\text { Prof.d'immersion de la } \\
\text { source en mer }\end{array}$ & $\begin{array}{c}\text { Profondeur d'immersion } \\
\text { en cuve }\end{array}$ & $\begin{array}{c}\text { Fréquences d'émission en } \\
\text { cuve }\end{array}$ & Fréquences transposées \\
\hline $200 \mathrm{~m}$ & $1 \mathrm{~cm}$ & $1 \mathrm{MHz}$ & $50 \mathrm{~Hz}$ \\
$1000 \mathrm{~m}$ & $5 \mathrm{~cm}$ & & $*$ \\
$2000 \mathrm{~m}$ & $10 \mathrm{~cm}$ & & $*$ \\
\hline
\end{tabular}


La courbe 1 montre la superposition du calcul [4] et de la mesure du niveau de pression en fonction de la distance source-récepteur dans de l'eau douce pour un fond plat. La source et le récepteur sont à 200 $\mathrm{m}$ ( $1 \mathrm{~cm}$ en cuve) de profondeur. La courbe 2 montre une mesure seule tracée dans les mêmes conditions que la courbe 1 mais avec un fond en pente. Alors que pour le fond plat le calcul et la mesure du champ de pression sont tout à fait comparables on voit dans la deuxième courbe que l'influence de la pente est forte surtout lorsque le plan incliné se rapproche de la surface quand la distance augmente.

courbe 1

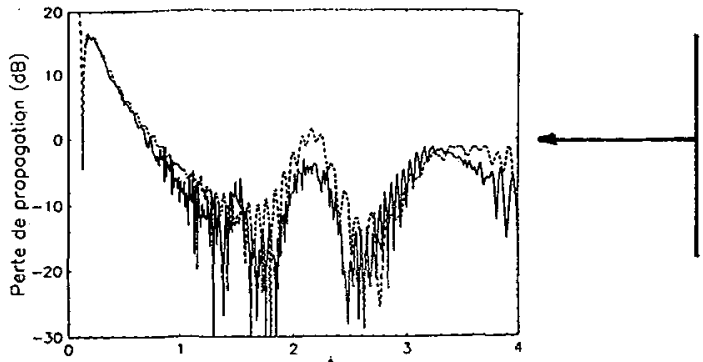

Niveau de pression mesuré en fonction de la distance sourcerécepteur - fond incliné (5\%) Emetteur et récepteur à $200 \mathrm{~m}$ de la surface $(1 \mathrm{~cm}$ en cuve)
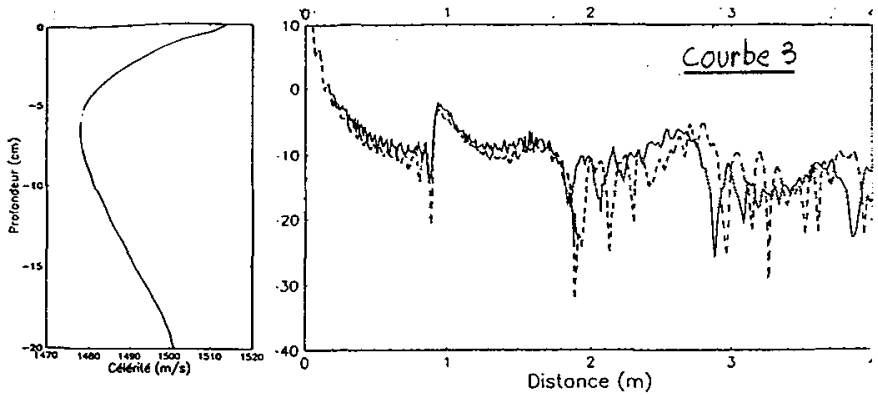

Niveau de pression en fonction de la distance source-récepteur en eau douce et sur un fond plat

Emetteur et récepteur à $200 \mathrm{~m}$ de la surface $(1 \mathrm{~cm}$ en cuve)

- - - niveau calculé niveau mesuré

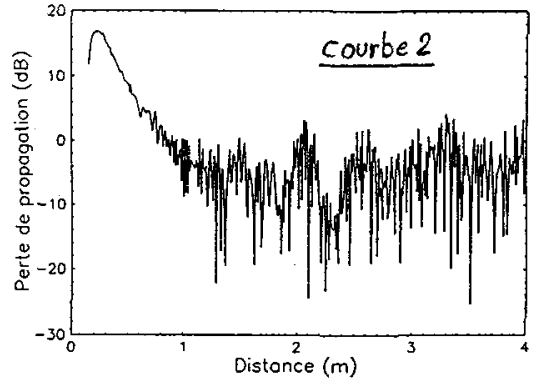

Niveau de pression en fonction de la distance source-récepteur en présence d'un profil vertical de célérité et sur un fond plat

Emetteur et récepteur à $1000 \mathrm{~m}$ de la surface $(6,5 \mathrm{~cm}$ en cuve)

- . - - niveau calculé niveau mesuré

De la même manière que pour la courbe 1 , la courbe 3 est le tésultat de la superposition du calcul et de la mesure du niveau de pression en fonction de la distance source-récepteur pour la source et le récepteur placés à $1000 \mathrm{~m}$ de la surface $(6,5 \mathrm{~cm}$ en cuve) pour un fond plat en présence d'un profil vertical de célérité

\section{CONCLUSION}

De nombreux calculs et mesures ont été faits en présence d'un profil bathycélérimétrique ou en eau douce. Dans tous les cas les résultats sont bons et la comparaison théorie-expérience tout à fait satisfaisante. Cela nous permet d'envisager la poursuite de cette étude en faisant varier tous les paramètres auxquels nous avons accés, à savoir la nature du fond (stratifié fluide-élastique), la pente (variation autour des $5 \%$ et passage d'un fond plat à un fond en pente) et le profil bathycélérimétrique.

\section{BIBLIOGRAPHIE}

[1] A.N. BARKHATOV, "Modelling of sound propagation in the sea"; Consultant Bureau. New York; London 1971

[2] G. RABAU, J. LEANDRE, R. HOLTZER, "Simulation d'environnement en acoustique sous marine", Journal de physique IV, Vol 2 , Colloque $n^{\circ} 1$, Suppl. JP III, $\mathrm{n}^{\circ} 4,2$ eme congrès francais d'acoustique.

[3] G. RABAU, "Modèle géoacoustique", Thèse de troisième cycle, Université d'Aix -MARSEILLE II, 1990

[4] D. LEE and S.T. McDANIEL, "Ocean Acoustic Propagation by Finite Difference Methods", Oxford : Pergamon Press, 1987 